\title{
Correction to: Ocular Oncology
}

\author{
Duangnate Rojanaporn
}

\section{Correction to: \\ Duangnate Rojanaporn (ed.), Ocular Oncology, https://doi.org/10.1007/978-981-13-2336-2}

In the original publication, the series title and the names of series editors on the cover and in the front matter pages had been inadvertently omitted. The series title "Retina Atlas" and the names of series editors (Sandeep Saxena, Richard F. Spaide, Eric H. Souied and Timothy Y.Y. Lai) have been included on the cover and the series title page in the front matter in the updated version. 